\title{
Mastery Learning of Toxicology Life Support Skills by ICU \& Emergency Toxicology Nurses using Simulation Technology in India
}

\author{
Verma $\mathbf{V}^{1 *}$, Shankar $\mathrm{S}^{2}$, Santosh $\mathrm{S}^{3}$, Joby $\mathrm{KO}^{4}$ and Jain $\mathrm{K}^{5}$ \\ ${ }^{1}$ Emergency \& Trauma care, Medanta-The Medicity, India \\ ${ }^{2}$ Nursing, Medanta-The Medicity, India \\ ${ }^{3}$ Nursing Incharge, Medanta-The Medicity, India \\ ${ }^{4}$ ICU Nursing Supervisor, Medanta-The Medicity, India \\ ${ }^{5}$ Nursing Preceptor, Medanta-The Medicity, India \\ ${ }^{6}$ Emergency Nursing Supervisor, Medanta-The Medicity, India
}

*Corresponding author: Dr Vivekanshu Verma, Associate consultant, Emergency \& Trauma care, Medanta-The Medicity, Gurugram, India, Email: vivekanshu@yahoo.co.in

\begin{abstract}
Simulation has been widely adopted as a training and assessment tool in nursing education on management of medical cases. Conventional teaching methods may be inadequate to adequately train healthcare providers for rare but potentially lethal events in Nursing such as managing Poisoning, associated polytrauma, Acute Brain stroke, Birthing process of a pregnant mother in labor, acute coronary syndrome and cardio-respiratory arrest due to poisoning or drug I I overdose. We have observed that Simulation based training, by enhancing provider skills, can subsequently decrease medical errors and increase patient safety.
\end{abstract}

Keywords: Poisoning; Simulation; Training; Resuscitation; Toxicology; Emergencies

\section{Introduction}

Our Hospital have created in-house simulation center with international training centre for the purpose of educating consultants and resident doctors, as well as Nurses and paramedics in BLS, ACLS, PALS, ISTOLS course for managing toxicology and various trauma management certified courses for fixed duration on regular basis, to update their skills and knowledge. Medical simulation offers the challenge of integrating medical knowledge, attitudes, and skills in case scenarios, procedure-based learning activities, and role playing [1].

Mannequins come in various shapes and sizes and can serve different purposes, including replication of the clinical deterioration and imitation of an adult in distress secondary to acute medical event. Fidelity describes the extent to which the appearance and behavior of the mannequin imitate the appearance and behavior of an actual patient. Simulation based training, by enhancing 
provider skills, can subsequently decrease medical errors and increase patient safety [2]. Simulation is especially effective in developing skills in procedures that require eye-hand coordination and ambidextrous maneuvers, such as securing airway in compromised airway due to vomiting and aspiration in unconscious state, vascular access in cardiovascular collapse, shock and cardiac arrest and the use of laryngoscopes for endotracheal intubation in respiratory compromise [3].

\section{Study Modules Analysis}

Nurses who receive simulator training performed better on actual patients, and Nurses performed better after receiving high-fidelity simulator training. Improvement in skills was demonstrated at 6-8 weeks, although many of these skills returned to baseline at 6-8 months. This finding suggests that teaching simulation is a skill that can be acquired across various levels of training regularly. This study emphasizes the importance of simulation training of nurses at regular intervals to ensure knowledge retention. Our hospital have integrated simulation exercises to better prepare their Nurses for rare or important (or both) medical conditions. Over the past five years, We have conducted several Simulation training in various aspects of nursing acute care training, evaluating the effectiveness of simulation as an educational tool, including

- Cardio pulmonary resuscitation.

- Trauma management.

- Airway management.

- Procedural skills in vascular access, Ryle's tube \& foley's catheterization.

- Crisis resource management/team-work training.

- "Code Yellow" disaster drill for mass casualty training.

- Toxicological emergencies.

- Birthing process via Obstetrics simulation.

- Code Blue \& Code Stoke simulation

\section{Code Blue Resuscitation}

Nursing acute care providers undergo formal resuscitation training (advanced Cardiac life support (ACLS), Pediatric advanced life support (PALS), and advanced trauma care for Nurses (ATCN)) every 2 years, yet a growing body of evidence suggests poor retention of these skills and knowledge. In addition, Nursing trainees are exposed to fewer critically ill patients during their training. Simulation training may potentially fill this educational void and improve the resuscitation skills of nursing healthcare professionals [4]. It has been demonstrated that Nurses who received additional training on a human patient simulator performed significantly better on the ACLS, PALS, ATCN written examination as well as during a mock resuscitation [5].

Pre-test and post-test analysis reported a mean improvement in overall performance by nursing residents after implementation of simulator training sessions. These novice learners in nursing demonstrated improved triage and intervention scores after each virtual reality disaster drill.

Nurses who received human patient simulator training in addition to ACLS showed significantly higher adherence to American Heart Association (AHA) standards as compared with those who received ACLS alone.

It was observed that the prior simulation training positively impacted their nursing skills during the resuscitation, including rapid problem recognition, correct choice and dosage of specific therapy, and coordination of team efforts.

\section{Trauma Management}

Trauma management has the potential to benefit from simulation training, as it is a highly stressful, relatively uncommon disease entity [6]. The advanced trauma Care Nursing (ATCN) training course, for emergency and trauma nurses, is offered every 4 years, but only a small component is devoted to simulation. Consequently, we developed training programs of "trauma code management" adopting simulation training to reinforce the various skills needed to manage acute trauma. Our training for nurses included multiple sessions of simulated trauma code followed by a debriefing and didactic session, and simulation training; randomized Nursing trainees to receive $10 \mathrm{~h}$ of trauma education by scenario-based didactic sessions and scenario based simulator sessions. Nursing trainees described a selfreported improvement in management of acute trauma, understanding roles, familiarity with the resuscitation room, and comfort with procedural skills after implementing a simulation-based educational session for nurses. Majority of Nurses reported significant improvement in overall team performance after simulation training in trauma code management. One possible explanation for this finding may have been the inclusion of nursing trainees in the simulated scenarios, which is often cited as a vital aspect of mock code training. A second trauma scenario performed 6 months later under "Code Yellow for mass disaster management" 
to evaluate retention of skills and knowledge resulted in significantly improved performance by emergency department nurses. After training, the simulation group had higher performance scores for crisis management skills, specifically with regard to teamwork.

Computer-based simulators: Screen-based simulation allowed nurses to participate in decision making scenarios at their own pace, with an instructor. These programs covered a wide range of topics, from basic science to trauma and bioterrorism. Patient-actors (standardized patients): They were trained Nurses who participate in medical scenarios. They contributed to the evaluation of the student's history taking, understanding of the physical examination, and interpersonal and communication skills.

\section{Airway Management}

Although basic airway management is a major component of resuscitation, it has been reported that Nurses lack basic airway skills. We examined the impact of a basic airway skills simulation program and found that simulation-trained Nurses performed significantly better on most elements of basic airway management during mock codes. Trained Nurses also showed improved performance on actual patients during a 6-month followup period. In addition to mastering basic airway skills, management of the 'difficult airway' is critical for nursing acute care providers in emergency. We evaluated difficult airway skills (laryngeal mask airway (LMA) intubation, endotracheal intubation guided via bougie) of Nurses after a simulation-based educational intervention. Improvement in skills was demonstrated at 6-8 weeks, although many of these skills returned to baseline at 6-8 months. This emphasizes the importance of training at regular intervals to ensure knowledge retention.

We compared 'low-fidelity' versus 'high-fidelity' simulation on nurses' ability to successfully perform nasogastric and urinary catheter placement. The lowfidelity mannequin consisted of a relevant body part model that allowed for tube insertion, in contrast to the high-fidelity simulator (anatomically correct mannequin that reacts to tube insertion with physical responses such as change in vital signs, gagging/coughing sounds, etc.). Nurses who received high fidelity training scored significantly higher than Nurses trained by the lowfidelity simulator. Nurses were subsequently evaluated on their peripheral line placement technique on critically ill adult patients. The 'course' group outperformed the 'traditional' group on the majority of clinical aspects of venous catheterization, including fewer attempts to find the vein, identification of anatomical landmarks, and total overall performance score. The 'course' group also scored higher on a multiple-choice test, supporting a correlation between knowledge gain and improved clinical performance.

\section{Crisis Resource Management/Team Training}

Leadership is often cited as a key aspect of Crisis resource management (CRM), which is a method of team training that focuses on behavioral skills, resource utilization, communication, leadership, and teamwork [7]. These skills are essential for effective clinical care, yet few nursing personnel are exposed to formal training in these areas. Team training has been found to decrease medical errors. Simulation offers an ideal setting to practice methods of CRM in a safe learning environment. Team training evaluated the effectiveness of a mock code-based educational intervention on the leadership skills of nurses, who participated in the training session displayed significantly improved leadership skills compared with nurses who did not undergo training. We conducted a specific program to teach simulation-based team training to nurses. These nurses completed a scenario-based course on team training using a computer-enhanced mannequin and standardized patients. Although the effect on clinical outcomes was not measured, nurses' feedback was universally positive.

Another training evaluated nursing-physician collaboration during three case scenarios using a human patient simulator. A panel of experts measured levels of nurse-physician collaboration using validated scoring systems. Physicians and nurses showed increased levels of collaboration and competency during the third scenario after working together for the first two simulation scenarios.

\section{Code Stroke Simulation}

We organized a stroke simulation that consisted of four stations dedicated to different aspects of acute stroke care. Two of the stations were high fidelity simulations in the setting of an emergency room and the other two of the stations were skill stations. During this simulation training Nurses were able to demonstrate the ability to lead a stroke code, demonstrate efficiency in delivery of acute stroke care, distinguish stroke from stroke mimics based on clinical features, performed a neurologic exam using NIH Stroke Scale in less than 5 minutes, gathered relevant information (historical, clinical, laboratory, imaging) in order to make a treatment determination, 
weighed indications and contraindications of thrombolytic (tPA) administration, identified the appropriate clinical context for interventional procedures, learned to take Consent from a patient/family for acute stroke intervention and formulated a plan for disposition and further management of stroke patients. All Nurses agreed that the overall quality and utility of debriefing sessions were good. Approximately $84 \%$ agreed that the simulation experience was more useful than reading a chapter or attending a lecture about the clinical scenario. After completing the sessions, all surveyed nurses agreed that they will be able to better facilitate future acute stroke care as compared to before the training. For the stroke code simulation session, there was a remarkable improvement between pre and post simulation scores i.e. (increased from 33\% to 87\%) with regards to agreement in feeling comfortable running a stroke code as a first responder from neurology service.

\section{Code Yellow Disaster Drill in Mass Casualty Training}

Our Institution conducted mass casualty drills every 6 months for nurses, incorporating actors and moulage, to prepare for mass casualty events. Our post drill analysis concluded that disaster training increases familiarity with procedures, identifies components of response, and provides opportunity to improve disaster response. Nursing Participants in the drills agreed that simulators and moulage closely mimicked real-life scenarios, accurately represented disease states, were effective at conveying images of injured victims and heightened realism of patient assessment and treatment. This drill helped to identify several critical errors made by first responders, including incorrect triage, inability to assess number of victims, and failure to notify concerned specialty. These nurses demonstrated improved triage and intervention scores after each subsequent virtual reality disaster drill.

\section{Toxicology Life Support Simulation}

Simulation is especially effective in developing skills in procedures that require eye-hand coordination and ambidextrous maneuvers, such as securing airway in compromised airway due to vomiting and aspiration in intoxicated state, vascular access in cardiovascular collapse, shock and cardiac arrest and the use of laryngoscopes for endotracheal intubation in respiratory Asphyxiants \& laryngeal angioedema due to anaphylactic reaction after exposure to toxins derived from insects, reptiles and fishes. Simulation training prepares learners to deal with unforeseen medical events in unknown poisoning, improves teamwork and communication skills among bedside doctors and nurses, and increases confidence and performance of healthcare providers in managing fatal toxic exposures. ISTOLS- Indian Society of Toxicology Life support course, helped to identify several critical errors made by first responders, including incorrect triage, inability to assess critically ill poisoned victims, and failure to resuscitate intoxicated patients in cardiac arrest. Participants agreed that the virtual reality simulator was effective at conveying images of intoxicated victims (Figure 1 ).

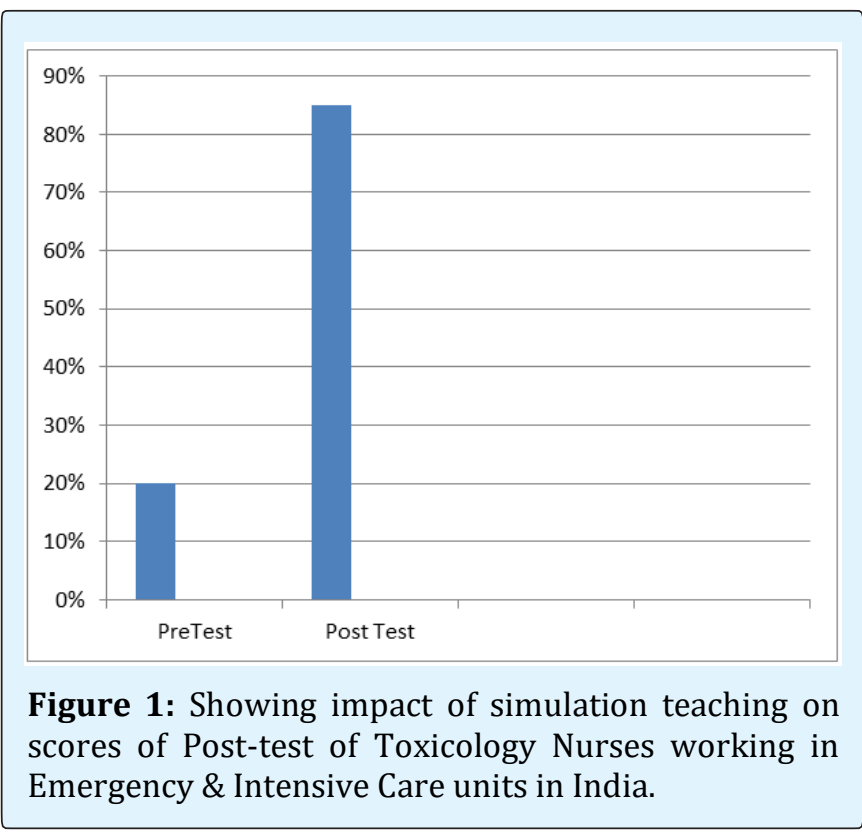

During recent toxicology life support workshop and training of 150 Toxicology Nurses working in Emergency \& Intensive Care units, held at Medanta the Medicity, Gurugram on $27^{\text {th }}$ November 2019, organized by Indian Society of Toxicology in collaboration with Indian Nursing Society, We observed that $94 \%$ of Toxicology Nurses working in Emergency \& Intensive Care units in India benefitted from increased confidence and perceived technical and non-technical skills during toxicological emergencies following simulation based learning. These novice learners in clinical toxicology demonstrated improved triage and intervention scores after each virtual reality toxidrome drill. Toxicology Nurses working in Emergency \& Intensive Care units in India, who received human patient simulator training in addition to advanced life support showed significantly higher adherence to international standards as compared with those who received basic life support alone. It was observed that the prior simulation training positively impacted their clinical 
skills during the resuscitation, including rapid problem recognition, correct choice and dosage of specific therapy, and coordination of team efforts. The differences of percentage in doctors' responses towards life support skills with and without the implementation of simulation practice were calculated and recorded. After the implementation of simulation training, Toxicology Nurses working in Emergency \& Intensive Care units showed more anticipation in the implementation of simulation training by hands-on practice on manikins and there was an increase from $63.89 \%$ to $89.44 \%$. Among Toxicology Nurses working in Emergency \& Intensive Care units, $65.35 \%$ of them liked life support skills as a subject, even before the simulation training were implemented. After the application of the simulation in training, $95.49 \%$ of residents were interested to learn life support skills in managing poisoning patients. There was an increase of $30.14 \%$ of Toxicology Nurses working in Emergency \& Intensive Care units, who had developed their liking towards life support skills in management of toxicological emergencies.

\section{Birthing process via Obstetrics Simulation}

Midwifery and emergency paramedical staff attending obstetrics participated in a simulation workshop where they were required to manage birth emergencies in real time with limited availability of resources to suit the setting. They completed a pre-test and post-test evaluation form exploring the content and utility of the workshops. Nurses underwent simulation training for assisting in vaginal delivery in vertex presentation, vaginal delivery via forceps \& urgent Caesarian section and managing post partum uterine bleeding on adult pelvic mannikins with infant mannikins with large head stuck in birth canal. Much of this training centered on sensitive tasks (i.e., pelvic examinations in females) or those that involve potential risk of harm to pregnant patients (securing airway and endotracheal intubation in cardiac arrest during child delivery or other invasive procedures like forceps delivery). Interprofessional teams of Nurses managed a simulated unplanned vaginal birth, followed by debriefing. Clinical knowledge was significantly increased in Nurses. All students were highly satisfied with the interprofessional simulation experience simulation. Birth simulation workshop was found to be a useful tool by Nurses that provide care to women who are undergoing childbirth [8]. Developing clear communication and teamwork were found to be the key learning principles guiding their practice.

\section{Conclusion}

There is ample evidence that simulation-based educational interventions in training Toxicology Nurses working in Emergency \& Intensive Care Units increase retention of knowledge for cardiopulmonary resuscitation, stroke code management, trauma \& toxicology care, airway management, procedural skills, team-training, and disaster management.

\section{Conflict of Interest: Nil}

\section{References}

1. Grady JL, Kehrer RG, Trusty CE, Entin EB, Entin EE, et al. (2008) Learning nursing procedures: the influence of simulator fidelity and student gender on teaching effectiveness. J Nurs Educ 47(9): 403-408.

2. (2005) Joint Commission (JCI) on Accreditation of Healthcare Organizations. Health Care at the Crossroads: Strategies for Improving the Medical Liability System and Prevention Patient Injury.

3. Bond WF, Lammers RL, Spillane LL, Smith-Coggins R, Fernandez R, et al. (2007) The use of simulation in emergency medicine: a research agenda. Acad Emerg Med 14(4): 353-363.

4. Kneebone R (2005) Evaluating clinical simulations for learning procedural skills: a theory-based approach. Acad Med 80(6): 549-553.

5. Steadman RH, Coates WC, Huang YM, Matevosian R, Larmon BR, et al. (2006) Simulation-based training is superior to problem-based learning for the acquisition of critical assessment and management skills. Crit Care Med 34(1): 151-157.

6. Brydges R, Carnahan H, Rose D, Rose L, Dubrowski A (2010) Coordinating progressive levels of simulation fidelity to maximize educational benefit. Acad Med 85(5): 806-812.

7. Issenberg SB, McGaghie WC, Petrusa ER, Lee Gordon D, Scalese RJ (2005) Features and uses of high-fidelity medical simulations that lead to effective learning: a BEME systematic review. Med Teach 27(1): 10-28.

8. Kumar A, Nestel D, Stoyles S, East C, Wallace EM, et al. (2016) Simulation based training in a publicly funded home birth programme in Australia: A qualitative study. Women Birth 29(1): 47-53. 